\section{Hemiballismus from cerebellar metastases}

Sir: A 50 year old alcoholic woman with metastatic mucinous signet ring cell adenocarcinoma of the colon developed irregular movements of the proximal and, to a lesser extent, distal joints of her right arm and leg. The next day, the movements became more abrupt, of wide-amplitude and almost continuous. They worsened when she became anxious during the neurological examination and disappeared when she slept. Her face and tongue were spared. Aside from generalised muscle weakness and hypoactive muscle stretch reflexes, her neurological examination was normal. She had no ataxia or terminal tremors of her limbs, nystagmus, hypotonia, slurred speech or abnormal mental function. She died of cardiorespiratory arrest two days later. At necropsy, she had a large metastatic tumour in her right cerebellar hemisphere impinging on the dentate nucleus and stretching the superior cerebellar peduncle (fig) and a smaller metastasis in the folia of the left cerebellar hemisphere. No gross or microscopic tumour was found in the decussation of the superior

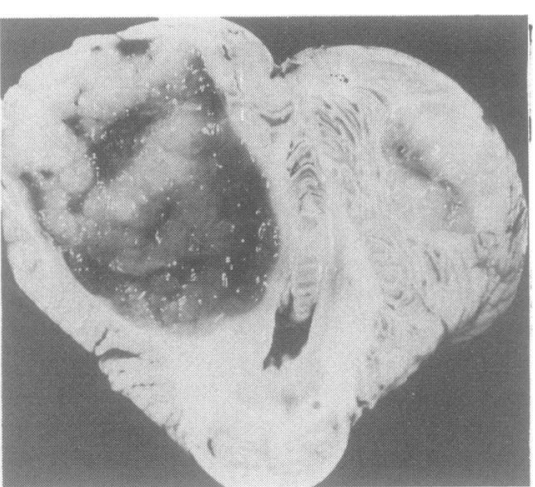

Fig Transverse section of the cerebellum and pons with large metastatic carcinoma on the right impinging on the dentate nucleus and stretching the superior cerebellar peduncle and a smaller metastasis in the periphery of the left hemisphere.

cerebellar peduncle, red nucleus or subthalamic nucleus of Luys. The latter was sectioned throughout its rostrocaudal extent on both sides. Our patient and others with nळ lesions in their subthalamus at necropsy' of on neuroimaging ${ }^{2}$ and the few patients witt subthalamic lesions but no hemiballismus remind us that the aetiopathogenesis of hemiballismus is still unexplained. Also, we should no longer teach that hemiballismus necessarily results from a lesion in the suber thalamus.

MARC G REYEढ़ EDWARD T STEVEN Division of Pathology: Cook County Hospitaks Chicago, IL 60612, USA

\section{References}

1 Schwarz GA, Barrows LJ. Hemiballism witR out involvement of Luys' body. Arch Neur\% 1960;2:420-34.

2 Srinivas K, Rao VM, Subdulaskshmi $\vec{P}$ Bhaskaran J. Hemiballism after striatat hemorrhage. Neurology 1987;37:1428-9. $\vec{\omega}$

3 Cooper IS. Involuntary Movement Disorder? New York: Harper and Row, 1969.

Accepted 28 October 1988

\section{Matters arising}

\section{A neuromuscular transmission disorder: com- bined myasthenia gravis and Lambert-Eaton syndrome in one patient}

Sir: In a recent article, Neuromuscular transmission disorder: combined myasthenia gravis and Lambert-Eaton syndrome in one patient Taphoorn' described a case with clinical and neurophysiological characteristics that suggested an association between the two diseases. The neurophysiological data were compatible with Lambert-Eaton myasthenic syndrome (LEMS) whereas some clinical observations indicated myasthenia gravis (MG). In another report antibodies antiacetylcholine receptors and anti-striated muscle antibodies were present in the serum ${ }^{2}$ as well as antibodies against thyroid and gastric mucosa; however, this finding is not an absolute criterion in differential diagnosis, since in both LEMS and MG associations with other autoimmune diseases have been reported. ${ }^{3}$

We observed a patient who died of an unidentified cause after presenting electromyography (EMG) findings and a clinical picture suggestive of a mixed form of LEMS and MG. The patient was a 61 year old man who had a 2 month history of myalgia, weakness of the pelvic and shoulder girdles with muscular exhaustibility on effort, dysphonia and dysphagia, slight bilateral ptosis of the eyelids, dry mouth and constipation. The symptoms had a recurrent pattern, each lasting several days, with abrupt onset and slowly progressive resolution.

The main symptoms reported by the patient (bilateral ptosis of the eyelids, impairment of the bulbar musculature, fluctuations of the symptoms) suggested MG whereas two EMGs performed during relapses were compatible with LEMS. The latter examinations demonstrated signs of myopathy of the proximal muscles and the first compound muscle action potential (CMAP) evoked by ulnar nerve stimulation had a small amplitude with an about $300 \%$ increase of the CMAP at repetitive stimulation at $30 \mathrm{~Hz}$. Administration of edrophonium chloride did not modify the clinical characteristics or EMG findings, nor was any benefit obtained from treatment with pyridostigmine bromide, performed also for diagnostic purposes. Screening for autoantibodies was negative, as were studies performed to demonstrate possible neoplastic disease (bone scintigraphy, computed tomography and magnetic resonance imaging of the mediastinum).

While the patient was still in hospital but in fairly good clinical condition and not receiving any treatment, on waking $\underset{\mathbb{Q}}{\mathbb{Q}} \boldsymbol{\theta}$ morning he had a sudden dyspnoeic cimis, became cyanotic and died in a few minutes Necropsy did not reveal any possible cofse of death, although a small-cell bronchial carcinoma of small size was found lymph node invasion.

The manner of death and the absence of documented cause seemed to us strongle suggestive of a myasthenic crisis, which has been reported in the literature in only three patients with LEMS; ${ }^{45}$ in these cases there was a progressive weakness, most marked the shoulder and pelvic girdles, and scant involvement of the ocular and bulbar mus cles. In these patients also death occurreg suddenly and "unexpectedly".45

We conclude that there may be a more frequent association between MG and LEMS than reported in the literature. The clinical and neurophysiological characteriso tics of the "overlap myasthenic syndrome should be better defined with the aim of clarifying its pathogenesis and because of possible therapeutic implications.

AMBROGIO BRENNA,

NATALE CURTÒ

DOMENICO D'URSO?

FRANCA D'AVANZO

*Division of Anatomical and Histologic® Pathology and Cytolog S. Gerardo Hospitax

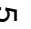

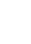


Monza, Italy.

†Division of Neurology,

S. Gerardo Hospital, Monza, Italy.

$\ddagger$ Division of Neurophysiology, Niguarda-Cà Granda Hospital, Milan, Italy.

§Division of Anaesthesiology, Niguarda-Cà Granda Hospital, Milan, Italy.

\section{References}

1 Taphoorn MJB, Van Duijn H, Wolters ECH. A neuromuscular transmission disorder: combined myasthenia gravis and Lambert-Eaton syndrome in one patient. $J$ Neurol Neurosurg Psychiatry 1988;51:880-2.

2 Tabbaa MA, Leshner RT, Campbell WW. Malignant thymoma with dysautonomia and disordered neuromuscular transmission. Arch Neurol 1986;43:955-7.

3 Lennon VA, Lambert EH, Whittingham S, Fairbanks V. Autoimmunity in the LambertEaton myasthenic syndrome. Muscle Nerve 1982;5:S21-S25.

4 Gutmann L, Crosby TW, Takamori M, Martin JD. The Lambert-Eaton syndrome and autoimmune disorders. Am J Med 1972;53: 354-6.

5 Castaigne P, Cambier J, Masson M, Cathala HP, Pierrot-Deseilligny E. Le syndrome pseudo-myasthenique paraneopolasique de Lambert-Eaton. Ann Med Int (Paris) 1969;120:313-22.

\section{Taphoorn et al reply:}

We thank Ambrogio and colleagues for their comments on our paper.'

They describe a patient with features, as they argue, of both myasthenia gravis and Lambert Eaton myasthenic syndrome (LEMS). The neurophysiological data recorded are compatible with LEMS, as was so in our patient. The combination, however, of LEMS and myasthenia gravis in the patient reported on is less evident than in our patient and in several patients described in the literature, for no antibodies against acetylcholine receptors were detected in serum. . $^{-5}$

The only features suggesting myasthenia gravis are the fluctuating oculobulbar symptoms, which are not exclusive for myasthenia gravis. ${ }^{6}$ Moreover we doubt if the manner of death, explained by the authors as a myasthenic crisis, really adds to the diagnosis myasthenia gravis in this patient.

We do not believe the so called "overlap myasthenic syndrome" to be a separate clinical entity; it merely is a combination of the two auto-immune diseases (LEMS and myasthenia gravis) in one patient.

As to the therapeutic implications, patients with a combination of LEMS and myasthenia gravis may be treated with corticosteroids, effective in both diseases. ${ }^{78}$ Our patient, on a $\mathbf{4 0} \mathrm{mg}$ prednisone alternate day dose, is still in a good clinical condition.

\section{References}

1 Taphoorn MJB, van Duijn H, Wolters ECh. A neuromuscular transmission disorder: combined myasthenia gravis and Lambert Eaton syndrome in one patient. $J$ Neurol Neurosurg Psychiatry 1988;51:880-2.

2 Fettel MR, Sin HS, Penn AS, Lovelace RE, Rowland LP. Combined Lambert Eaton syndrome and myasthenia gravis. Neurology 1978;28:398.

3 Singer P, Smith L, Ziegler DK, Festoff BW. Post-tetanic potentiation in a patient with myasthenia gravis. Neurology 1981;31: 1345-7.

4 Tabbaa MA, Leshner RT, Campbell WW. Malignant thymoma with dysautonomia and disordered neuromuscular transmission. Arch Neurol 1986;43:955-7.

5 Oh SJ, Dwyer DS, Bradley RJ. Overlap myasthenic syndrome: combined myasthenic gravis and Lambert Eaton syndrome. Neurology 1987;37:1411-4.

6 Kula RW. Facilitating myasthenic syndrome. In: Vinken PJ, Bruyn GW. Handbook of Clinical Neurology. Amsterdam: North Holland 1979;41:349-61.

7 Brunner NG, Namba T, Grob D. Corticosteroids in management of severe, generalized myasthenia gravis. Neurology 1972; 22:603-10.

8 Streib EW, Rothner AD. Lambert Eaton myasthenic syndrome: long-term treatment of three patients with prednisone. Ann Neurol 1981;10:448-53.

\section{Trial of ganglioside GM1 in acute stroke}

Sir: In their therapeutic trial, recently reported in this Journal, Dr Hoffbrand and colleagues did not find GM1 therapy "to be of value in the treatment of acute stroke".' This indication seems in contrast with two previous clinical studies, ${ }^{23}$ but the differences in the experimental designs, which are expression of two different approaches concerning the use of GM1 in stroke, may explain the discrepancies of the results.

In our trial ${ }^{2}$ GM1 treatment was started two weeks after the onset of neurological deficits (when antioedema therapy had already been stopped) and it was continued daily for six weeks. At the end of this period the naturally occurring recovery after stroke was significantly enhanced by the drug. In our opinion GM1 seems to play a role in functional recovery by stimulating the adap- tative reorganisation and the complex mechanisms of the neuronal plasticity (that is neuronal sprouting).

Hoffbrand and colleagues started GM1 therapy within 72 hours from the onset of the neurological deficits and continued for four weeks. It seems to us that such an approach might eventually show the antioedema effectiveness of GM1 (no other antioedema drugs were mentioned), but this drug effect has still to be demonstrated. Furthermore, this treatment could not clearly evaluate GM1 role in enhancing functional recovery because therapy was stopped too early.

Nevertheless, it must be pointed out that Hoffbrand's study reveals two data which are in partial accordance with our findings: (1) as regards mortality, the prognosis is better for patients on therapy with active drug: nine patients in placebo group and five patients in GM1 group died in Hoffbrand's trial, while in our study three patients in placebo and no one in GM1 group died (see patients and methods section of our paper); (2) in Hoffbrand's study, the mean increase in Barthel Index from the end of the first to the sixth month was 7.7 for the placebo group (from 72.3 to 80.0 ) and 18.0 for the GM1 group (from $70 \cdot 2$ to 88.2): probabl such a wide difference is not statisticall 9 significant because the study group is nog sufficiently large and homogeneous.

On the whole, these data indicate a possible effectiveness of GM1 in the treatment of stroke. We think that the real efficacy of the drug will be demonstrated not only by $\overrightarrow{0}$ larger study, but also through more selective criteria regarding the stroke (ischaemic or haemorrhagic) and the time of therapy (beginning and duration).

I APPOLLONIO

L FRATTOLA M MASSAROTTI* Neurological Clinic, University of Milan, San Gerardo Hospital, Monza, Italy. * Fidia Research Laboratories, Abano Terme, Italy.

Address correspondence to: Professor Frattola Lodovico, Clinica Neurologica, Ospedale San Gerardo, Via Donizetti, 106, 20052 Monza (MI), Italy.

\section{References}

1 Hoffbrand BJ, Bingley PJ, Oppenheimer SM, Sheldon CD. Trial of ganglioside GMI in acute stroke. J Neurol Neurosurg Psychiatry 1988;51:1213-4.

2 Bassi S, Albizzati MG, Sbacchi M, Frattola L, Massarotti M. Double blind evaluation of 